\title{
Neurofeedback training of auditory selective attention enhances speech-in-noise perception
}

Abbreviated title: selective attention training

\section{Subong Kim, ${ }^{1}$ Caroline Emory, ${ }^{1}$ and Inyong Choi ${ }^{1,2, *}$}

${ }^{1}$ Department of Communication Sciences and Disorders, University of lowa, lowa City, IA 52242, USA

2 Department of Otolaryngology - Head and Neck Surgery, University of lowa Hospitals and Clinics, lowa City, IA 52242, USA

subong-kim@uiowa.edu, caroline-emory@uiowa.edu, inyong-choi@uiowa.edu

* Corresponding author at: Department of Communication Sciences and Disorders, University of lowa, 250 Hawkins Dr., lowa City, IA 52242, USA. Email address: inyongchoi@uiowa.edu 


\section{Abstract (100 words limit)}

1 Selective attention enhances cortical responses to attended sensory inputs while

2 suppressing others, which can be an effective strategy for speech-in-noise ( $\mathrm{SiN}$ )

3 understanding. Here, we introduce a training paradigm designed to reinforce attentional

4 modulation of auditory evoked responses. Subjects attended one of two speech

5 streams while our EEG-based attention decoder provided online feedback. After four

6 weeks of this neurofeedback training, subjects exhibited enhanced cortical response to

7 target speech and improved performance during a SiN task. Such training effects were

8 not found in the Placebo group that underwent attention training without neurofeedback.

$9 \quad$ These results suggest an effective rehabilitation for $\mathrm{SiN}$ deficits.

10

Keywords: selective attention, neurofeedback training, speech-in-noise perception 
Albeit it is crucial for effective communication, the ability to understand speech in

14 noise (SiN) differs dramatically even across normal-hearing individuals (Kumar et al.,

15 2007; Moore et al., 2013). One reason for poor SiN understanding can be the

16 deteriorated selective attention (Bressler et al., 2017), as past studies showed a

17 correlation between selective attention and SiN performance (Strait and Kraus, 2011).

18 Compared to poor performers, listeners with good SiN performance exhibit greater

19 amplitude ratio of cortical responses to target speech compared to responses to noise

20 (Kim et al., 2019), which may indicate that attentional modulation on neural encoding of

21 acoustic inputs in the auditory cortex (AC) (Hillyard et al., 1973; Mesgarani and Chang,

22 2012; Carcea et al., 2017) is a key neural mechanism for successful SiN understanding.

23 Conventional hearing remediations through amplification cannot improve SiN

24 understanding ability (Bentler et al., 2008). Instead, perceptual training is often

25 considered as a solution for SiN difficulties (Whitton et al., 2014; Whitton et al., 2017).

26 However, a frequently reported problem of perceptual training is that the training effect

27 does not generalize to other auditory stimuli not used for the training (Fiorentini and

28 Berardi, 1981; Wright et al., 1997). This generalization problem leads us to consider a

29 training that directly improves a key strategy for the SiN understanding: a training that

30 reinforces attentional modulation of auditory cortical responses.

How, then, can we design a perceptual training paradigm that aims to reinforce

32 attentional modulation of cortical activity? Theories of learning claim that the target of

33 training is manipulated by rewarding; the determination of feedback (i.e., reward or

34 punishment) must be based on the target training component (Goodman and Wood, 
2004). Thus, to enhance attentional modulation of cortical responses, a training paradigm should provide feedback based on the strength of attentional modulation. Auditory selective attention can be reliably decoded from single-trial electroencephalographic (EEG) signals (Kerlin et al., 2010; Choi et al., 2013; O'Sullivan et al., 2015). Since the accuracy of attention decoding from single-trial EEG signals reflects the strength of attentional modulation on cortical auditory evoked responses

41 (Choi et al., 2013), providing the result of EEG-based attention decoding as

42 neurofeedback (Sherlin et al., 2011) may reinforce the users' attentional modulation of

43 cortical responses. The goal of the present study is to provide evidence to support the

44 concept of auditory selective attention training through such an EEG-based

45 neurofeedback paradigm and explore its efficacy for SiN understanding ability.

\section{Methods}

\section{Participants}

Twenty young adult subjects with normal hearing, who were native speakers of American English, were recruited for this study (Mean age $=23.2$ years; $S D=1.33$ years; $6(30 \%)$ male). Upon agreeing to the study, subjects were randomly assigned to

51 either the Experimental or the Placebo (control) group (i.e., single-blinded design). All

52 subjects completed four consecutive weeks of one-hour-per-week training and pre- and

53 post-training $\mathrm{SiN}$ tests at their first and last visits. All study procedures were reviewed

54 and approved by the University of lowa Institutional Review Board.

\section{Experimental design and procedures}

$56 \quad$ Attention training procedure: Experimental group 
During each training session, three overlapping auditory streams were presented; 1) a male voice saying the word "down" repeated four times from the right

59 (+30 azimuth) loudspeaker, 2) a female voice saying the word "up" repeated five times

60 from the left $\left(-30^{\circ}\right)$ loudspeaker, 3) and a distractor non-speech noise that sounds like

61 water bubble played intermittently from the loudspeaker directly in front of the subject.

62 For each of the 120 trials in each visit, a visual cue ("Target: Up" or "Target: Down") was

63 given to direct participants' attention to either "up" or "down" stream (60 trials each).

64 After sounds, listeners' attention was decoded from EEG. A visual feedback ("+" sign on

65 the screen moving up or down) was given at the end of a trial to indicate the decoded

66 direction of attention (i.e., attended "up" or "down" stream, respectively). Figure 1

67 illustrates an example of a trial attending the "down" stream.

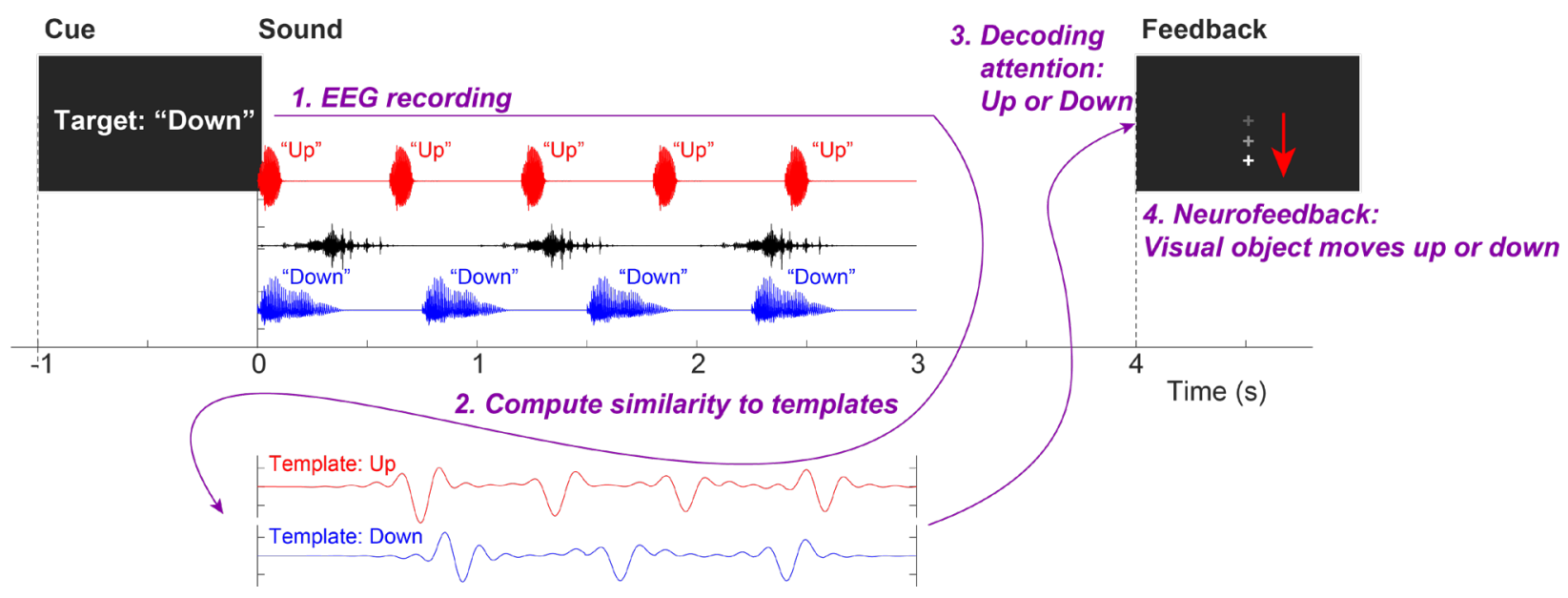

69 Figure 1. Trial structure of the neurofeedback training assigned to the Experimental

70 group. This example shows an attend-down trial.

$71 \quad$ Attention training procedure: Placebo group

73 (i.e., isochronous repetitions of "up" and "down" spoken by the female and male 
74 speakers) where one of the last 3 or 2 utterances in each stream had 3-semi-tone

75 higher pitch. As the visual cue directed their attention to either "up" or "down" stream in

76 each trial, they picked an utterance with a higher pitch in the attended stream by

77 pressing number key (i.e., an oddball detection task). After the button press, visual

78 feedback ("Correct" or "Incorrect") was given based on the accuracy of their button

79 response.

Pre- and post-training speech-in-noise tests

All subjects, regardless of group designation, completed the same pre- and post-

82 training SiN test. The test used 100 monosyllabic consonant-vowel-consonant English

83 words from a pre-recorded California Consonant Test with added multi-talker babble

84 noise. Stimuli were presented at $\pm 3 \mathrm{~dB}$ SNR (50 words each) by changing the level of

85 the noise in random order. At each trial, a target word started 1 second after the noise

86 onset. At the end of a trial, subjects picked a word they heard from four choices given

87 on the screen. For further analysis, behavioral and neural data from $-3 \mathrm{~dB}$ SNR

88 condition showing larger individual differences and no ceiling effect were used.

\section{ERP analysis}

Sixty-four channel scalp EEG data were recorded during the training and SiN

91 tasks using the BioSemi ActiveTwo system at a $2048 \mathrm{~Hz}$ sampling rate with the

92 international $10-20$ configuration. In order to provide neurofeedback to the Experimental

93 group, a template-matching method was used to decode attention from single-trial EEG

94 signals (Choi et al., 2013). EEG recordings from front-central channels (Fz, FCz, FC1,

$95 \mathrm{FC} 2, \mathrm{Cz}$ ) were averaged and re-referenced to linked mastoids. EEG signals were 
96 bandpass-filtered between 1 and $9 \mathrm{~Hz}$, baseline corrected, and then compared to two

97 pre-generated template EEG waveforms obtained from cortical evoked responses to

98 "up" and "down" streams. The attention was decoded by finding the template that has a

99 larger correlation coefficient with the EEG signal. For analyzing EEG data obtained

100 during SiN tasks, after applying a bandpass filter between 1 and $30 \mathrm{~Hz}$ using a 2048-

101 point FIR filter, epochs were extracted and baseline-corrected. After correcting ocular

102 artifacts using independent component analysis (Jung et al., 2000), the epochs were

103 averaged at each electrode. Since we use non-repeating naturally spoken words as

104 stimuli, the latency of event-related potentials (e.g., N1) varied across words. To obtain

105 clean N1 from averaged evoked response, every epoch was rearranged according to

106 the median N1 latency of its corresponding word obtained from the grand mean of 50

107 normal hearing subjects who completed the same SiN task in our laboratory previously.

108 In order to project the sensor-space data into source-space, the inverse operator

109 was estimated using minimum norm estimation (MNE) (Hämäläinen, 1989; Gramfort et

110 al., 2013; Gramfort et al., 2014) based on assumptions of multiple sparse priors (Friston

111 et al., 2008) on an average template brain. To focus on non-speech-specific attention

112 effect on AC while avoiding confounding factors related to language processing, we

113 chose right - instead of left - Heschl's gyrus (HG) as the region of interest.

\section{Statistical analysis}

Two-way mixed ANOVAs were conducted on both behavioral performance and

116 neural data. To get clear ERPs and perform statistical analysis on neural data, we

117 computed leave-one-out grand averages (i.e., jackknife approach). In addition, ERP

118 envelopes were extracted by applying a $4-8 \mathrm{~Hz}$ bandpass filter and Hilbert transform 
119 for ERP magnitude comparisons. The peak magnitudes of ERP envelopes obtained at

$120 \sim 230$ ms after the word onset from the right HG were compared between conditions. As

121 post hoc analysis, one-tailed $t$-tests were conducted with Bonferroni correction as we

122 were only interested in the improvement (i.e., one direction) in the behavioral

123 performance (accuracy) and acoustic encoding at AC after training. Inflated F-ratios and

$124 t$-values due to the jackknife approach were adjusted (Luck, 2014).

\section{Results}

\section{Behavioral performance}

We found that the behavioral performance (accuracy) did not improve after training $\left[F_{1,18}=3.37, p=0.083\right]$ on average; however, accuracy did increase significantly in the Experimental group [ $\left.t_{9}=-2.37, p=0.042\right]$, but not in the Placebo group [tg $=-0.71, p=0.49]$ (Figure 2A). We found no group effect $\left[F_{1,18}=2.49, p=\right.$ 0.13] on average; however, the post hoc analysis showed a significant difference in

132 performance between two groups after training [ $\left.t_{18}=2.15, p=0.045\right]$, but not before 133 training $\left[t_{18}=0.60, p=0.56\right]$ (Figure 2A). No interaction between training and group

$134 \quad\left[F_{1,18}=0.38, p=0.55\right]$ was revealed.

\section{Attention during training}

During the attention training, both Experimental and Placebo groups showed a

137 significant effect of attention on evoked responses as reflected in the single-trial

138 attention decoding accuracy being higher than chance level (one-sample $t$-test,

139 Experimental group: $t_{9}=8.34, p<0.001$, Placebo group: $\left.t_{9}=5.12, p<0.001\right)$. The

140 mean accuracy of single-trial attention decoding was $59.1 \%$ and $58.1 \%$, with a standard 
141 deviation of $3.5 \%$ and $5.0 \%$ for the Experimental and Placebo groups, respectively.

142 There was no significant difference in the single-trial classification accuracies between

143 the groups (two-sample t-test, $p=0.59$ ). The mean accuracy of the oddball detection in

144 the Placebo group was $98.8 \%$, with a $1.4 \%$ standard deviation.

\section{Source-space ERPs to SiN}

147 response to $\mathrm{SiN}$ increased significantly after training $\left[F_{1,18}=4.78, p=0.042\right]$; notably, 148 the training effect only appeared in the Experimental group [tg $=-3.16, p=0.023$ ], but 149 not in the Placebo group $\left[t_{9}=-0.14, p=1\right]$ (Figure 2D). The $F$-test results revealed no 150 group effect $\left[F_{1,18}=1.94, p=0.18\right]$ on average; the post hoc analysis showed no 151 significant difference in $\mathrm{AC}$ response to $\mathrm{SiN}$ between two groups before $\left[t_{18}=-0.41, p=\right.$ 152 1] and after training $\left[t_{18}=2.024, p=0.12\right]$ (Figure 2D). There existed no significant 153 interaction between training and group $\left[F_{1,18}=3.89, p=0.064\right]$.
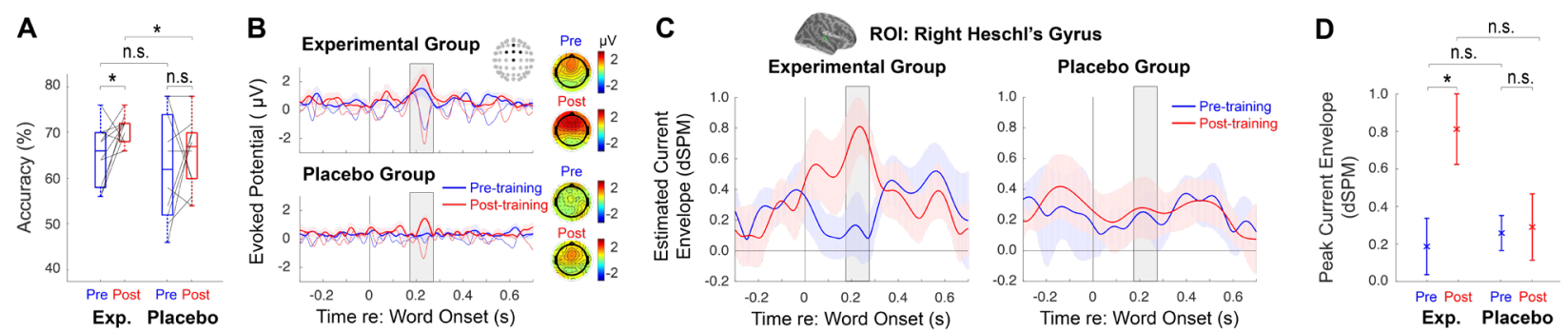

Figure 2. Training effects on A. accuracy, B. sensor-space evoked responses (thick

156 lines: envelopes) and topographies, C. source time courses (current envelopes with \pm 1

157 standard error) in the right HG, and D. the peak evoked magnitude in the right HG. A

158 significant training effect on the peak evoked magnitude is found in the Experimental 159 group. 
This study demonstrated that the neurofeedback training of auditory selective attention is effective for improving neural encoding and accurate speech recognition in background noise. As it is evident that attentional gain control process (Hillyard et al.,

164 1998) is involved in a successful speech-in-noise perception (Mesgarani and Chang,

165 2012; Kim et al., 2019), our training paradigm was designed to reinforce attentional

166 modulation of auditory cortical evoked potentials by providing visual feedback

167 determined by an EEG-based attention decoder. After four weeks of training, we found 168 consistent improvement in listeners' SiN performance and acoustic encoding at AC only 169 in the Experimental group.

171 control for the Experimental group after training (Shinn-Cunningham and Best, 2008).

172 This is consistent with the previous finding that showed attention could modulate the

173 sound representation in AC and improve behavioral performance (Mesgarani and

174 Chang, 2012; Carcea et al., 2017). Attention may increase the gain at the neural

175 population level by increasing the response of neurons to the target word. Learning or

176 training can improve selective enhancement of neural response, that may develop over

177 time and last longer, and improve speech perception (Froemke et al., 2013). The

178 training effect found in the present study may develop and last over weeks and result in 179 an improvement in speech perception.

180 Our Experimental group training was differentiated from the Placebo training in 181 that the feedback reward was determined by participants' neural activity, not by 182 behavioral performance. Similarly to the reports by Whitton et al. (2014) and Whitton et 
183 al. (2017), the present study showed that the effect of neurofeedback training was

184 transferred to SiN performance, while the Placebo group did not show such

185 generalizability. The generalizability of the training effect observed in the present study

186 may indicate that the reinforcement of attentional modulation on cortical responses may

187 improve a key neural strategy for the SiN understanding. In contrast, our Placebo

188 training provided a primary task of detecting pitch oddballs from the attended stream.

189 Feedbacks provided to the Placebo group informed how their oddball detection was

190 accurate, not how strong their attention was. Our results may indicate the "indirect"

191 primary task that does not provide a reward shaped by the strength of attention would

192 not exhibit generalizability of its training effect to the speech in noise task.

\section{Acknowledgments}

194 This work was supported by American Otological Society Research Grant and

195 Department of Defense Hearing Restoration Research Program Grant (W81XWH-19-1-

196 0637) awarded to Inyong Choi, as well as NIDCD P50 (DC000242 31). The authors

197 declare no conflicting financial interests. 


\section{References}

200

201

202

203

204

205

206

207

208

209

210

211

212

213

214

215

216

217

218

219

220

221

222

223

224

225

226

227

228

229

230

231

232

233

234

235

236

237

238

239

240

241

242

243

244

245

246

247

Bentler, R., Wu, Y. H., Kettel, J., and Hurtig, R. (2008). "Digital noise reduction: outcomes from laboratory and field studies," International journal of audiology 47, 447-460.

Bressler, S., Goldberg, H., and Shinn-Cunningham, B. (2017). "Sensory coding and cognitive processing of sound in Veterans with blast exposure," Hear Res 349, 98-110.

Carcea, I., Insanally, M. N., Froemke, R. C., Carcea, I., Insanally, M. N., and Froemke, R. C. (2017). "Dynamics of auditory cortical activity during behavioural engagement and auditory perception," Nat. Commun. Nature Communications 8.

Choi, I., Rajaram, S., Varghese, L. A., and Shinn-Cunningham, B. G. (2013). "Quantifying attentional modulation of auditory-evoked cortical responses from single-trial electroencephalography," Front Hum Neurosci 7, 115.

Fiorentini, A., and Berardi, N. (1981). "Learning in grating waveform discrimination: Specificity for orientation and spatial frequency," VR Vision Research 21, 1149,1153-1151,1158.

Friston, K., Harrison, L., Daunizeau, J., Kiebel, S., Phillips, C., Trujillo-Barreto, N., Henson, R., Flandin, G., and Mattout, J. (2008). "Multiple sparse priors for the M/EEG inverse problem," Neuroimage 39, 1104-1120.

Froemke, R. C., Carcea, I., Barker, A. J., Yuan, K., Seybold, B. A., Martins, A. R., Zaika, N., Bernstein, H., Wachs, M., Levis, P. A., Polley, D. B., Merzenich, M. M., and Schreiner, C. E. (2013). "Long-term modification of cortical synapses improves sensory perception," Nat Neurosci 16, 79-88.

Goodman, J. S., and Wood, R. E. (2004). "Feedback specificity, learning opportunities, and learning," The Journal of applied psychology 89, 809-821.

Gramfort, A., Luessi, M., Larson, E., Engemann, D. A., Strohmeier, D., Brodbeck, C., Goj, R., Jas, M., Brooks, T., Parkkonen, L., and Hamalainen, M. (2013). "MEG and EEG data analysis with MNE-Python," Front Neurosci 7, 267.

Gramfort, A., Luessi, M., Larson, E., Engemann, D. A., Strohmeier, D., Brodbeck, C., Parkkonen, L., and Hamalainen, M. S. (2014). "MNE software for processing MEG and EEG data," Neuroimage 86, 446-460.

Hämäläinen, M. S., Sarvas, J. (1989). "Realistic conductivity geometry model of the human head for interpretation of neuromagnetic data," IEEE Trans Biomed Eng 36, 165-171.

Hillyard, S. A., Hink, R. F., Schwent, V. L., and Picton, T. W. (1973). "Electrical signs of selective attention in the human brain," Science 182, 177-180.

Hillyard, S. A., Vogel, E. K., and Luck, S. J. (1998). "Sensory gain control (amplification) as a mechanism of selective attention: electrophysiological and neuroimaging evidence," Philosophical transactions of the Royal Society of London. Series B, Biological sciences 353, 1257-1270.

Jung, T. P., Makeig, S., Humphries, C., Lee, T. W., McKeown, M. J., Iragui, V., and Sejnowski, T. J. (2000). "Removing electroencephalographic artifacts by blind source separation," Psychophysiology 37, 163-178.

Kerlin, J. R., Shahin, A. J., and Miller, L. M. (2010). "Attentional gain control of ongoing cortical speech representations in a "cocktail party"," J Neurosci 30, 620-628.

Kim, S., Schwalje, A., Liu, A., Gander, P., McMurray, B., Griffiths, T., and Choi, I. (2019). "External and Internal Signal-to-noise Ratios Alter Timing and Location of Cortical Activities During Speech-in-noise Perception," bioRxiv 817460.

Kumar, G., Amen, F., and Roy, D. (2007). "Normal hearing tests: is a further appointment really necessary?," Journal of the Royal Society of Medicine Journal of the Royal Society of Medicine 100, 66.

Luck, S. J. (2014). An introduction to the event-related potential technique (The MIT Press, Cambridge, Massachusetts). 
Mesgarani, N., and Chang, E. F. (2012). "Selective cortical representation of attended speaker in multi-talker speech perception," Nature 485, 233-236.

Moore, D. R., Rosen, S., Bamiou, D.-E., Campbell, N. G., and Sirimanna, T. (2013). "Evolving concepts of developmental auditory processing disorder (APD): A British Society of Audiology APD Special Interest Group white paper," International journal of audiology 52, 3-13.

O'Sullivan, J. A., Power, A. J., Mesgarani, N., Rajaram, S., Foxe, J. J., Shinn-Cunningham, B. G., Slaney, M., Shamma, S. A., and Lalor, E. C. (2015). "Attentional Selection in a Cocktail Party Environment Can Be Decoded from Single-Trial EEG," Cerebral Cortex 25, 1697-1706.

Sherlin, L. H., Arns, M., Lubar, J., Heinrich, H., Kerson, C., Strehl, U., and Sterman, M. B. (2011). "Neurofeedback and Basic Learning Theory: Implications for Research and Practice," Journal of Neurotherapy 15, 292-304.

Shinn-Cunningham, B. G., and Best, V. (2008). "Selective attention in normal and impaired hearing," Trends Amplif 12, 283-299.

Strait, D. L., and Kraus, N. (2011). "Can you hear me now? Musical training shapes functional brain networks for selective auditory attention and hearing speech in noise," Front Psychol 2, 113-113.

Whitton, J. P., Hancock, K. E., and Polley, D. B. (2014). "Immersive audiomotor game play enhances neural and perceptual salience of weak signals in noise," procnatiacadscie Proceedings of the National Academy of Sciences of the United States of America 111, 9030.

Whitton, J. P., Hancock, K. E., Shannon, J. M., and Polley, D. B. (2017). "Audiomotor Perceptual Training Enhances Speech Intelligibility in Background Noise," Current biology : CB. 27, 3237-3247.e3236.

Wright, B. A., Buonomano, D. V., Mahncke, H. W., and Merzenich, M. M. (1997). "Learning and generalization of auditory temporal-interval discrimination in humans," The Journal of neuroscience : the official journal of the Society for Neuroscience 17, 3956-3963. 